\title{
Heterostructured Layer Growth of Polyaniline by Vacuum Thermal Evaporation and Fabrication of Thin-Film Capacitors
}

\author{
Shatrudhan Palsaniya ${ }^{\mathrm{a}}$, Harshal B. Nemade ${ }^{\mathrm{a}, \mathrm{b}, *}$ and Ashok Kumar Dasmahapatra, ${ }^{\mathrm{a}, \mathrm{c}, *}$ \\ ${ }^{\mathrm{a} C e n t r e}$ for Nanotechnology, Indian Institute of Technology Guwahati, Guwahati - 781039, \\ Assam, India. \\ ${ }^{\mathrm{b}}$ Department of Electronics and Electrical Engineering, Indian Institute of Technology \\ Guwahati, Guwahati - 781039, Assam, India.
}

${ }^{\mathrm{c}}$ Department of Chemical Engineering, Indian Institute of Technology Guwahati, Guwahati 781039, Assam, India.

\footnotetext{
* Corresponding authors: Phone: +91-361-2582273, Fax: +91-361-2582291, Email: akdm@iitg.ac.in (A. K. Dasmahapatra); Phone: +91-361-2582509, Fax: +91-361-2582542, E-mail: harshal@iitg.ac.in (H. B. Nemade)
} 


\section{S1. Analysis of spectroscopic ellipsometry for the optical constants}

Spectroscopic ellipsometry (SE) allows in-situ monitoring and indispensable analysis of nanostructured thin films. The SE measurements are capable to quantize the morphology, composition, interlayer diffusion, and surface interaction of thin films. The refractive index and film thickness are key parameters to reproduce the scientific results for various applications, especially for thin film devices. Thus, maintaining a film thickness is highly necessary to investigate the physical parameters, such as complex refractive index and dielectric constants, etc. The complex reflectance ratio $(\rho)$ of parallel $(\mathrm{P})$ and perpendicular $(\mathrm{S})$ plane polarized light waves defined as $R_{p} / R_{s}$, where, $R_{p}$ and $R_{s}$ are coefficients of reflection. The reflected light characterized by two distinct angles of $\Psi$ and $\Delta$ are correlated as follows ${ }^{1-}$ $3 ;$

$$
\tan (\Psi) \mathrm{e}^{\mathrm{i} \Delta}=\frac{\mathrm{R}_{\mathrm{p}}}{\mathrm{R}_{\mathrm{s}}}
$$

where, $\Psi$ is an amplitude ratio of the parallel plane incidence and perpendicular polarizations, and $\Delta$ is the phase difference. These parameters are strongly rely on film thickness. A twolayer model was used to evaluate thin films. The refractive index (n), and extinction coefficient (k) were extracted from the measured $\psi$ and $\Delta$ following the Tauc-Lorentz fitting model. The finest fitting of the experimental values is identified by minimizing the root mean square error (RMSE) as follows ${ }^{4}$;

$$
\text { RMSE }=\sqrt{\frac{1}{2 N-M_{i}} \sum_{i=1}^{N}\left[\left(\frac{\psi_{i}^{\text {Mod }}-\psi_{i}^{\text {Exp }}}{\sigma_{\psi, i}^{\operatorname{Exp}}}\right)^{2}+\left(\frac{\Delta_{i}^{\text {Mod }}-\Delta_{i}^{\text {Exp }}}{\sigma_{\Delta, i}^{\operatorname{Exp}}}\right)^{2}\right]}
$$

Where, $(\psi, \Delta)$ is sum of ' $N$ ' experimental values, $M$ signifies variable parameters, $\sigma$ is the standard deviation, Mod and Exp in superscript are attributed to modeled/or fitted and experimental values, respectively.

We report variable angle SE measurements in the visible range using the rotating analyzer. The SE measurements of single-layered PANI-ES $(96.5 \mathrm{~nm})$, PANI-EB $(68.8 \mathrm{~nm})$ and PANI- 
ES/PANI-EB heterostructure $(49.4 \mathrm{~nm})$ thin films were performed on glass substrates. We have recorded variable thickness due to a slight deviation in heating rate and deposition time. Thin film samples longitudinally mounted fixing three angles of incidences $\left(60^{\circ}, 65^{\circ}\right.$ and $\left.70^{\circ}\right)$ at the room temperature and $\mathrm{RH} \%$ 65. The values of $\Delta$ and $\Psi$ (Figure S1) are fitted maintaining a minimum value of the RMSE. A fractional deviation between measured and fitted data, is attributed to the interfacial region ${ }^{2,5}$ between PANI layers and substrate. Similarly, using the fitted optical constants, we have observed complex dielectric function (real and imaginary) for the PANI thin films (Figure S2). 

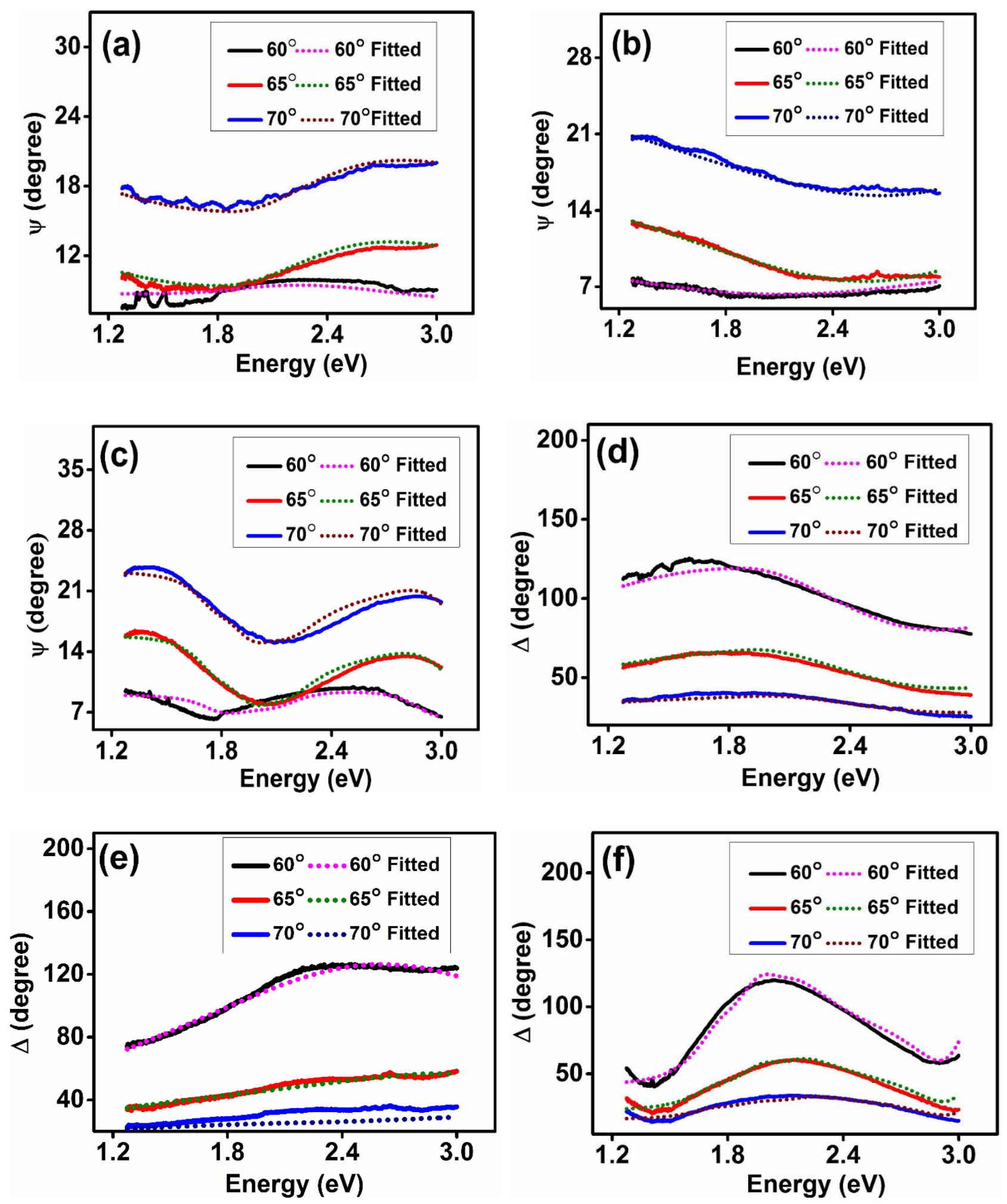

Figure S1. Spectroscopic Ellipsometry analysis of $\psi$ for (a) PANI-ES, (b) PANI-EB, and (c) PANI-ES/PANI-EB thin films. Similarly, $\Delta$ for (d) PANI-ES, (e) PANI-EB, and (f) PANIES/PANI-EB thin films. Thick and dotted lines are associated to measured and fitted graphs, respectively. 

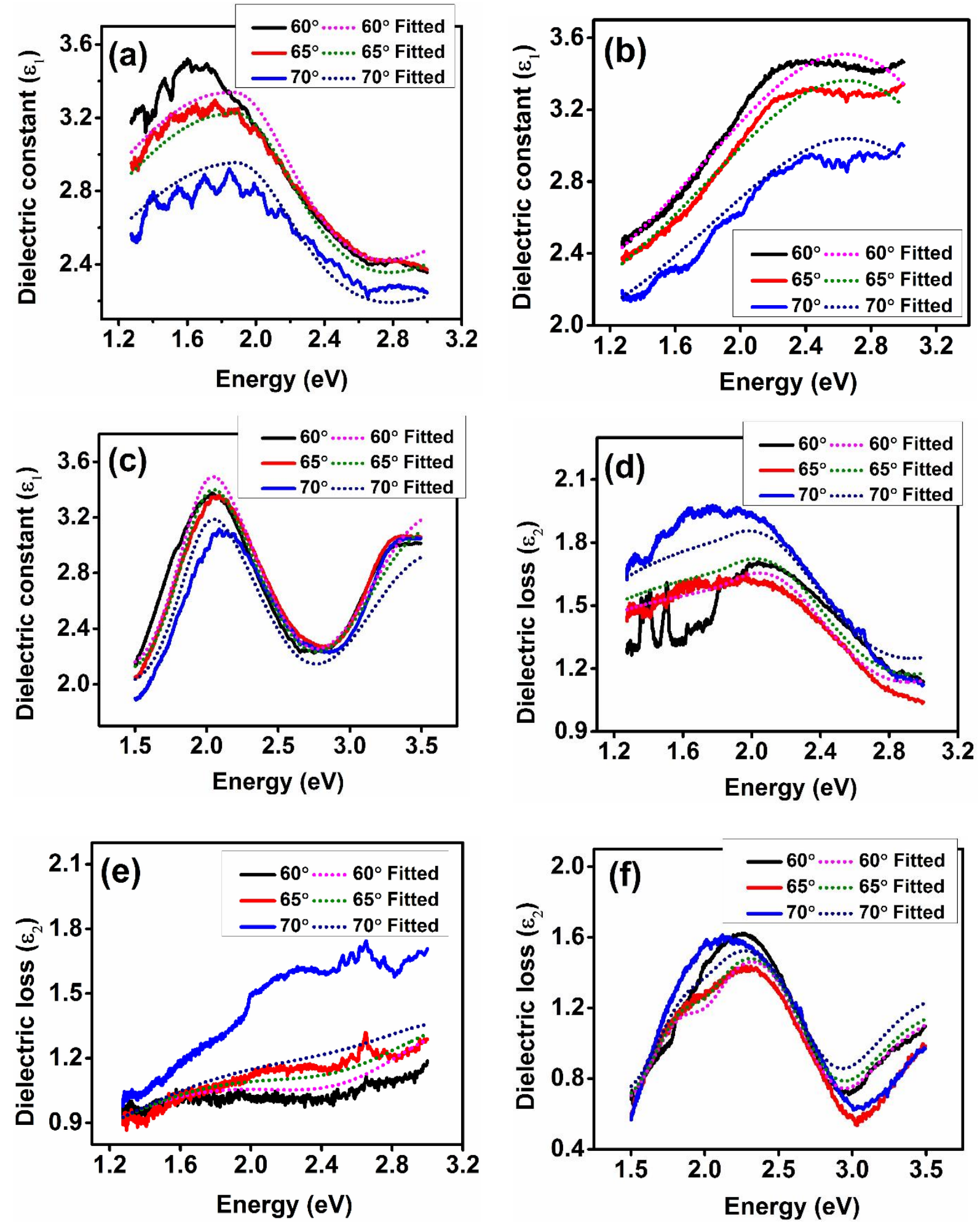

Figure S2. The real function of dielectric permittivity $\left(\varepsilon_{1}\right)$ for (a) PANI-ES, (b) PANI-EB, and (c) PANI-ES/PANI-EB thin films. Similarly, imaginary function of dielectric losses $\left(\varepsilon_{2}\right)$ for (d) PANI-ES, (e) PANI-EB, and (f) PANI-ES/PANI-EB are plotted with the photon energies. Thick and dotted lines are correspond to measured and fitted graphs, respectively. 


\section{S2. Electric field intensity of the uniform charged thin film}

The PANI thin film is equivalent to thin sheet having uniform surface charge density $\left(\sigma_{\mathrm{s}}\right)$, as schematically shown in Figure S3.

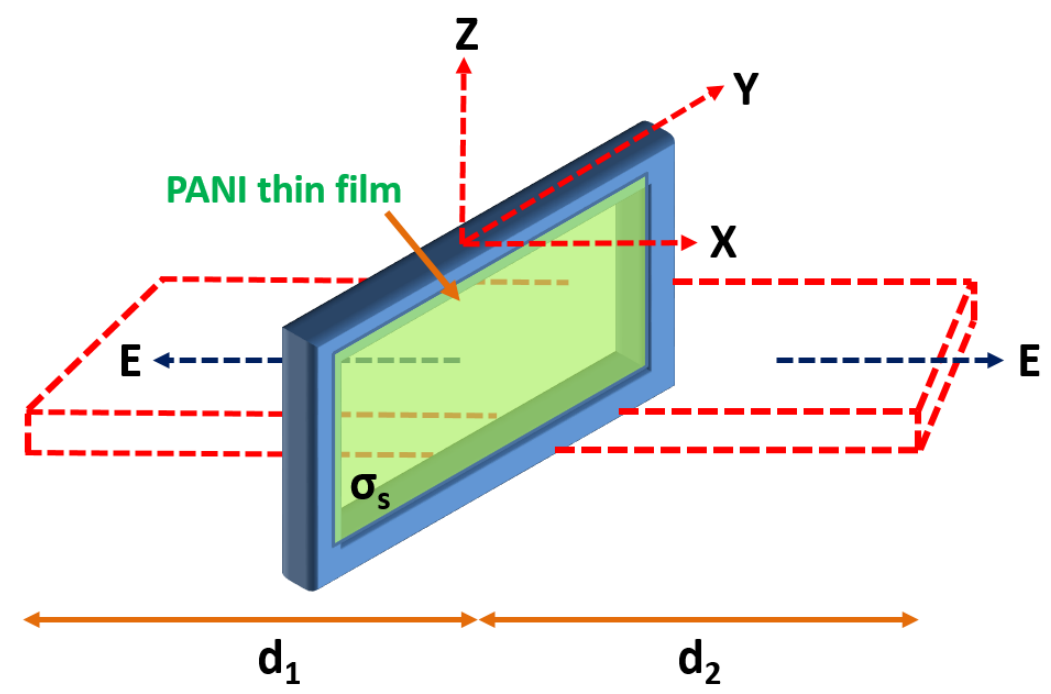

Figure S3. Schematic illustration of the electric field direction due to surface charge density of the PANI thin films.

Electric field lines passes through PANI film based on the electric field $(\overrightarrow{\mathrm{E}})$ can be analyzed as follows ${ }^{6}$ :

The above shown thin film region is considered to be a rectangular Gaussian surface. It is clearly seen that two faces with unit vector $\hat{\imath}$ would be alongside $\overrightarrow{\mathrm{E}}$. Hence, $\overrightarrow{\mathrm{E}}$ is perpendicular to the thin film throughout the surface area (A). The charge flux across the film is $\overrightarrow{\mathrm{E}} . \hat{\mathrm{i}} \mathrm{A}$. The net flux of the Gaussian surface from $-\mathrm{X}$ to $+\mathrm{X}$ directions is equivalent to that would be sum of 2EA, which is enclosed by the surface charge $q$. In another, $q=\sigma_{s} \times A$, where $\sigma_{s}\left(\mathrm{C} \mathrm{m}^{-2}\right)$ is the surface charge density. Therefore,

$$
2 \mathrm{EA}=\frac{\mathrm{q}}{\varepsilon_{0}}=\frac{\sigma_{\mathrm{s}} \mathrm{A}}{\varepsilon_{0}}
$$

Accordingly, the electric field intensity will be as follows:

$$
\mathrm{E}=\frac{\sigma_{\mathrm{s}}}{2 \varepsilon_{0}} \mathrm{~V} \mathrm{~m}^{-1}
$$


Here, $\sigma_{\mathrm{s}}\left(\right.$ Coulomb $\left.\times \mathrm{m}^{-2}\right)$ and $\varepsilon_{0}\left(\operatorname{Farad} \times \mathrm{m}^{-1}\right)$. For the electric field $\mathrm{E}$ is $\frac{\text { Coulomb }}{\text { Farad }} \mathrm{m}^{-1}$, and we know that 1 Farad $=1$ coulomb per volt. After estimation we have electric field in volt per meter.

\section{References:}

(1) Losurdo, M.; Bergmair, M.; Bruno, G.; Cattelan, D.; Cobet, C.; de Martino, A.; Fleischer, K.; Dohcevic-Mitrovic, Z.; Esser, N.; Galliet, M.; et al. Spectroscopic Ellipsometry and Polarimetry for Materials and Systems Analysis at the Nanometer Scale: State-of-the-Art, Potential, and Perspectives. J. Nanoparticle Res. 2009, 11, 1521-1554.

(2) Hinrichs, K.; Gensch, M.; Nikonenko, N.; Pionteck, J.; Eichhorn, K.-J. Spectroscopic Ellipsometry for Characterization of Thin Films of Polymer Blends. Macromol. Symp. $\mathbf{2 0 0 5}, 230,26-32$.

(3) Ogieglo, W.; Wormeester, H.; Eichhorn, K.-J.; Wessling, M.; Benes, N. E. In Situ Ellipsometry Studies on Swelling of Thin Polymer Films: A Review. Prog. Polym. Sci. $\mathbf{2 0 1 5}, 42,42-78$.

(4) Sirard, S. M.; Green, P. F.; Johnston, K. P. Spectroscopic Ellipsometry Investigation of the Swelling of Poly(Dimethylsiloxane) Thin Films with High Pressure Carbon Dioxide. J. Phys. Chem. B 2001, 105, 766-772.

(5) Ogieglo, W.; Wormeester, H.; Wessling, M.; Benes, N. E. Spectroscopic Ellipsometry Analysis of a Thin Film Composite Membrane Consisting of Polysulfone on a Porous $\alpha$ Alumina Support. ACS Appl. Mater. Interfaces 2012, 4, 935-943.

(6) Eskola, L. Elements of Electrostatics and Potential Theory. In Geophysical Interpretation using Integral Equations; Eskola, L., Ed.; Springer Netherlands: Dordrecht, 1992; pp 920. 